\title{
Nanofibers Based on Thermoplastic Elastomers and Isofural ${ }^{+}$
}

\author{
George Bogdan Spurcaciu 1, Laurentiu Marin 1,*, Rodica-Mariana Ion 1,2, Maria Rapa ${ }^{\text {, }}$ \\ Stefan Robu ${ }^{4}$, Paul Ghioca 1, Lorena Iancu 1,2, Ramona Grigorescu 1, Cristian Nicolae 1, \\ Mihaela Udrea ${ }^{1}$ and Carla Paduretu ${ }^{1}$
}

1 National Institute for Research \& Development in Chemistry \& Petrochemistry -ICECHIM, 202 Splaiul Independentei, 060021 Bucharest, Romania; bogdan.spurcaciu@icechim.ro (G.B.S.); rodica.ion@icechim.ro (R.-M.I.); paul.ghioca@icechim.ro (P.G.); lorena.iancu@icechim.ro (L.I.); ramona.grigorescu@icechim.ro (R.G.); cristian.nicolae@icechim.ro (C.N.); mihaela.udrea@icechim.ro (M.U.); carla.paduretu@icechim.ro (C.P.)

2 Doctoral School of Materials Engineering Department, Valahia University, 13 Aleea Sinaia, 130004 Târgovişte, Romania

3 University POLITEHNICA of Bucharest, Center for Research and Eco-Metallurgical Expertise, 313 Splaiul Independentei, 060042 Bucharest, Romania; rapa_m2002@yahoo.com

4 Moldova State University, 60 Mateevici str., MD-2009 Chişinău, Moldova; stefan_robu@yahoo.com

* Correspondence: laurentiu.marin@icechim.ro

† Presented at the 16th International Symposium "Priorities of Chemistry for a Sustainable Development" PRIOCHEM, Bucharest, Romania, 28-30 October 2020.

Published: 16 November 2020

Keywords: nanofibers; isofural; thermoplastic elastomers; antibacterial; electrospinning

The study aim was the synthesis and characterization by thermal and structural properties of nanofibers, obtained by electrospinning using styrene-butadiene block-copolymers (SBS) and styrene-isoprene block-copolymers (SIS), as well as their composites with isofural [1].

In the first step, styrene-butadiene block-copolymers (SBS) and styrene-isoprene block-copolymers (SBS) were obtained by anionic sequential polymerization. The reactions were carried out in cyclohexane solution through a three-stage process and were initiated with n-butyl lithium. In the second step, polymer composites with antibacterial properties were obtained, using the synthetized thermoplastic elastomers and isofural in tetrahydrofuran solution [2].

The polymeric composites with antibacterial properties obtained from thermoplastic elastomers and isofural were used for the manufacture of nanofibers by electrospinning (Figures 1 and 2).

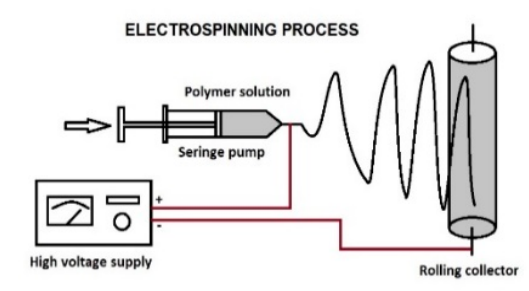

Figure 1. Schematic representation of electrospinning process. 


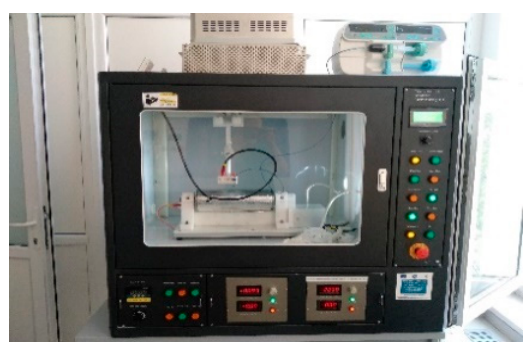

Figure 2. Electrospinning equipment.

The polymer nanofibers manufactured by electrospinning [3] were characterized by ATR-FTIR analysis, Differential Scanning Calorimetry (DSC), and Thermo-gravimetric Analysis (TGA).

The results indicated that the nanofibers obtained from composites of thermoplastic elastomers and isofural have a corresponding thermal stability. The thermal decomposition started after $330^{\circ} \mathrm{C}$ in the case of SBS and after $300^{\circ} \mathrm{C}$ in the case of SIS.

Acknowledgments: This paper was supported by a Nucleu Program, project no. PN.19.23.03.01, contract no. $23 \mathrm{~N} / 2019$.

\section{References}

1. Kakiage, M.; Oda, S. Nanofibrous hydroxyapatite composed of nanoparticles fabricated by Electrospinning. Mater. Lett. 2017, 248, 114-118.

2. Ghioca, P.; Iancu, L.; Grigorescu, R.; Spurcaciu, B.; Rapa, M.; Cincu, C.; Pica, A.; Matei, E. Recovered Polypropylene Composites with High Impact Strength. Mater. Plast. 2017, 1, 18-22.

3. Nagarajan, S.; Pochatbohatier, C.; Balme, S.; Miele, P.; Kalkura, S.N.; Bechelany, M. Electrospun fibers in regenerative tissue engineering and drug delivery. Pure App. Chem. 2017, 89, 1799-1808.

Publisher's note: MDPI stays neutral with regard to jurisdictional claims in published maps and institutional affiliations.

(C) 2020 by the authors. Licensee MDPI, Basel, Switzerland. This article is an open access article distributed under the terms and conditions of the Creative Commons Attribution (CC BY) license (http://creativecommons.org/licenses/by/4.0/). 